\title{
Osgood Schlatter disease: An Audit Profile of 35 Adolescents
}

Authors: Kireti V.M. MBChB, MMed(Surg) , Atinga J.E., MBChB, MMed, MSc(Ortho), Affiliation: Department of Orthopedic Surgery, University of Nairobi, Correspondence: Kireti, V.M, P. 0 Box 19741 Nairobi, 00202 - Nairobi, Email: vmkireti@yahoo.ca

\section{Abstract}

\author{
Background \\ Osgood-Schlatter disease is a common cause of anterior knee pain in \\ the adolescent. Treatment is usually conservative with surgery reserved \\ for those who do not respond to this treatment. There is little published \\ work regarding the experience with the disease in our local set up. This \\ series documents the experience with 35 adolescents treated for the \\ disease. \\ Design \\ Case series \\ Subjects \\ Thirty five adolescents with clinical and radiological diagnoses of \\ Osgood-Schlatter disease at Nairobi and Kenyatta National Hospitals, \\ between 2001and 2007. \\ Method \\ Patients were evaluated for demographics, knee involvement, activities
}

associated with pain and treatment outcome

Results

There were 28 males and 7 females, aged 10 to 16 years (mean 12.8 years). Thirteen had bilateral knee involvement. Twenty two were involved in active sports while the rest had constant pain and unable to sit or kneel. A family history of the disease was documented in one case. Thirty adolescents responded well to the conservative treatment. In the five adolescents who underwent surgery, the patella tendon was edematous with thickening of the tendon sheath and neovascularisation. All the operated adolescents returned to active sports with 6 weeks after the surgery.

Conclusion

Osgood-Schlatter disease is a self-limiting condition in majority of adolescents. Surgery when indicated has an excellent outcome.

\section{Introduction}

Osgood-Schlatter disease is a common cause of anterior knee pain in the adolescent. Paget first described the disease in 1891. Osgood and Schlatter in 1903 published separate papers in which they differentiated it from avulsion fractures of the tibial tubercle $(1,2)$. Diagnosis is based on the symptoms that include anterior knee pain made worse by exercise and relieved by resting the knee. Examination usually shows tenderness and edema of the tibial tubercle. X- Rays may show fragmentation of the tibial tubercle.

Osgood-Schlatter disease is typically a benign self-limiting condition but may take months to years to resolve completely. Symptomatic management of the pain involves rest of the knee, topical or systemic analgesics and rarely bracing or casting is necessary. Surgery is usually reserved for those who are not responding to the conservative regime and usual result is complete resolution of the symptoms. Surgery may also be performed for cosmetic reasons in cases of prominence of the tibial tubercle (2). This paper documents our experience with the disease in 35 adolescents over a 6 year period.

\section{Patients \& Methods}

This audit was conducted at the Nairobi Hospital and Kenyatta National teaching and referral hospital. The adolescents were treated for Osgood Schlatter disease between 2001 and 2007. The diagnosis was based on (i) anterior knee pain, (ii) tenderness over the tibial tubercle and a (iii) lateral knee X-ray showing tibial tubercle osteochondritis with or without fragmentation. The treatment protocol involved a discussion with the parents and the adolescents and included rest from the provoking activities, topical and oral analgesics and RICE 'principle' in acute pain. The patients were treated and followed up in the clinic for an average period of oneyear. Surgery was offered to those who failed to respond to conservative measures.

\section{Results}

Twenty eight males and 7 females were studied. The age ranged from $10-16$ years, with a mean age was 12.8 years.

Thirteen adolescents had bilateral knee involvement while 16 had left knee involvement (Fig. 1). Two of the 
patients were siblings. A family history was documented for another one case.

All 35 adolescents were not able to kneel. Sixteen had pain when bending the knee while eight had constant pain (Figure 2). Twenty two were involved in active sporting activities including football, basketball and running.

Thirty patients responded to conservative treatment. Symptoms escalated or did not resolve for five males aged between 13-16 years of age. The latter were therefore offered surgery. The radiographs in all the five cases showed loose ossicles which had not united (Fig. 3)

Surgical findings included oedematous tendons, increased vascular response from the medial and lateral sides of the tibial tubercle, blanching of tendon insertions, thickened tendon sheath and inflamed infrapatella bursa. The bony ossicles and inflamed bursae were excised (Fig. 3b). Histology confirmed the inflammatory changes.

\section{Discussion}

Osgood-Schlatter disease is a common cause of anterior knee pain in the adolescents. The disease presents bilaterally in about 25 percent of the cases and is usually associated with sporting activity (3-7). In this study a higher proportion (39\%) had bilateral knee involvement and $60 \%$ involved in some form of sports. Single injury, sporting activity or repeated flexing of the knee against a tight quadriceps muscle causing micro trauma to the tibial tuberosity is the probable mechanism. As shown in this audit however, some adolescents develop pain with normal activities like sitting, bending the knee and kneeling.

A radiological evaluation usually confirms the diagnosis and also rules out other causes of anterior knee pain that may include quadriceps tendon avulsion, patellofemoral stress syndrome, pes anserinus bursitis, chondromalacia patellae, osteomyelitis of the proximal tibia, Patellar tendonitis, and Sinding-Larsen-Johansson syndrome. In early stages, X-rays show irregularity of the tibial apophysis and separation from the tibial tubercle $(3,8)$. Later. fragmentation of the tibial tubercle occurs. These may be in two groups, proximal fragment(s) and distal fragment(s). The proximal fragment is usually tilted and attached to the patella tendon. The distal fragment heals but the proximal may persist as a loose fragment and may cause persistent pain and handicap that may need

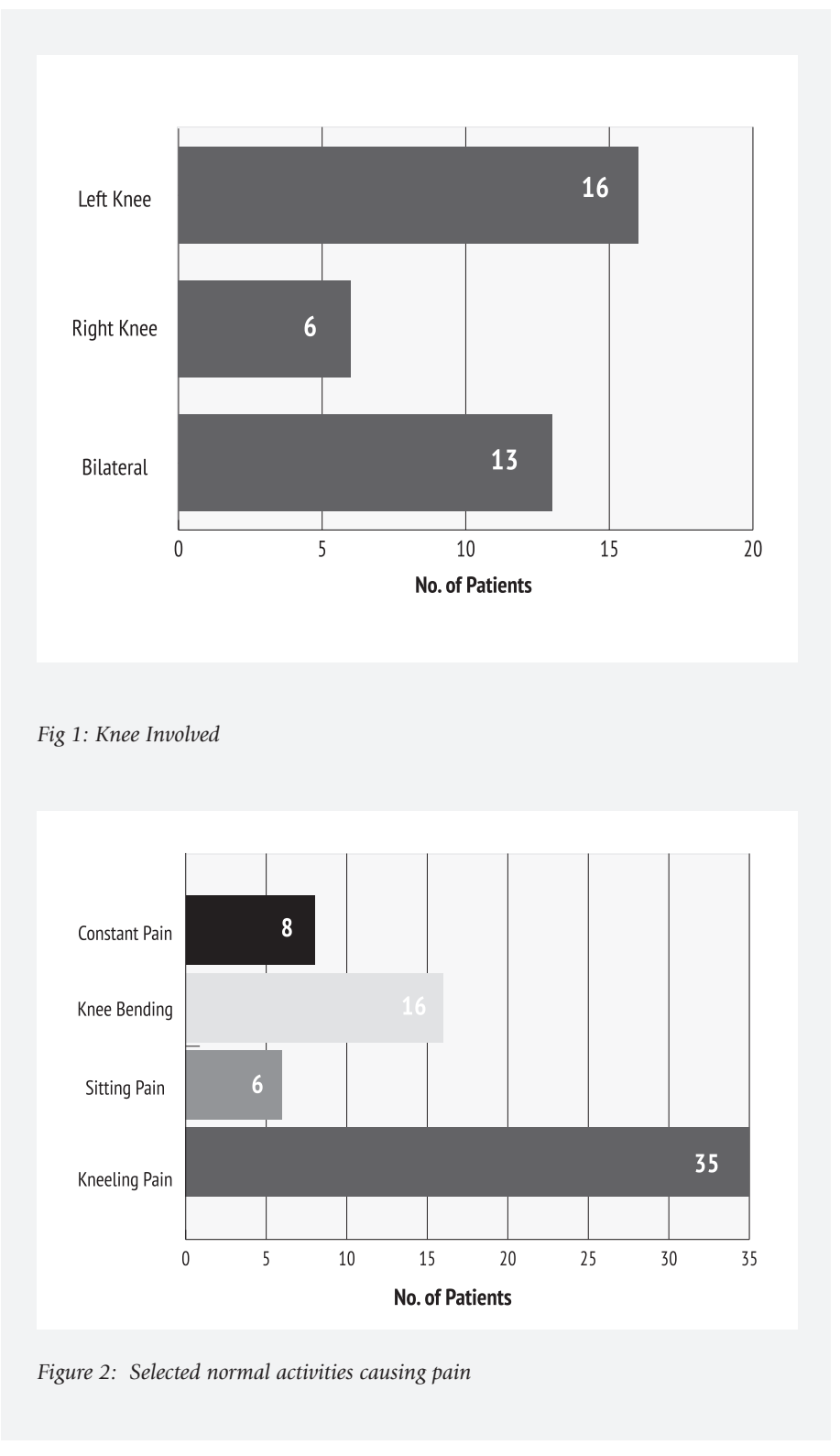

surgery $(3,8,9)$. Other investigations may include ultrasonograph, CT and MRI studies which may show abnormal size of the patella tendon, oedema of the tendon, distended infrapatella bursa and ossicles (10). Although no ultrasound, CT or MRI was performed in this study, the five adolescents who underwent surgery were found to have thickened tendon sheaths, oedema and thickening of the infrapatella bursa linings.

Osgood-Schlatter disease is a self-limiting condition, which normally heals without any residual deformity or disabilities and treatment is therefore conservative (310). In the acute phase, rest of the knee, ice packs and analgesics topically or systemically help to relieve the pain. 


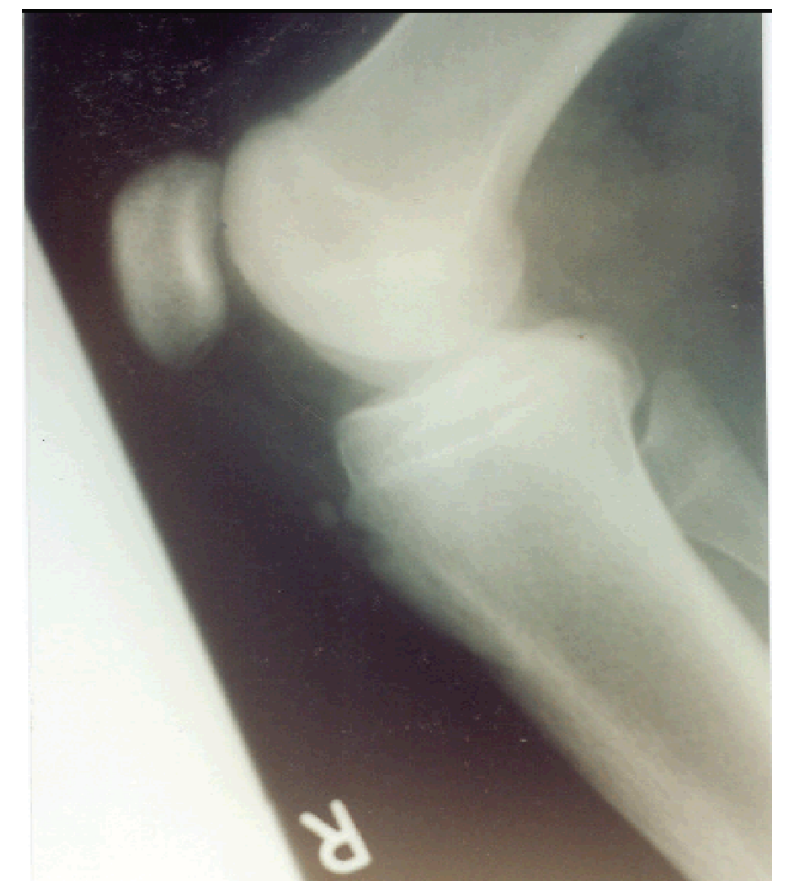

Fig 3a: Lateral knee radiograph of Osgood-Schlatter disease showing a loose ossicle

This may involve educating the adolescent of the need to avoid the activities that make the condition worse. For teenagers not compliant with this regime, it is recommended to protect the tuberosity with a knee brace or a plaster cast cylinder for 2-4 weeks. In this study, 30 of the teenagers responded well to the conservative treatment and returned to their normal activities within 1 year. Surgery has a very limited role in the treatment of Osgood-Schlatter disease. It is reserved for those who do not respond to the conservative treatment or have recurrent disabling pain $(11,12,13$,$) . In general, surgical$ intervention yields good results, especially for patients with bony or cartilaginous ossicles in which the ossicles are excised $(3,9,11)$. Infact the presence of a loose bony ossicle may be used as a pointer to surgical intervention. All the five patients subjected to surgery had symptom resolution on follow up. Other surgical procedures described for the disease include tibial tuberosity excision (14). Local steroid injection is not recommended for the treatment of the condition because of the associated side

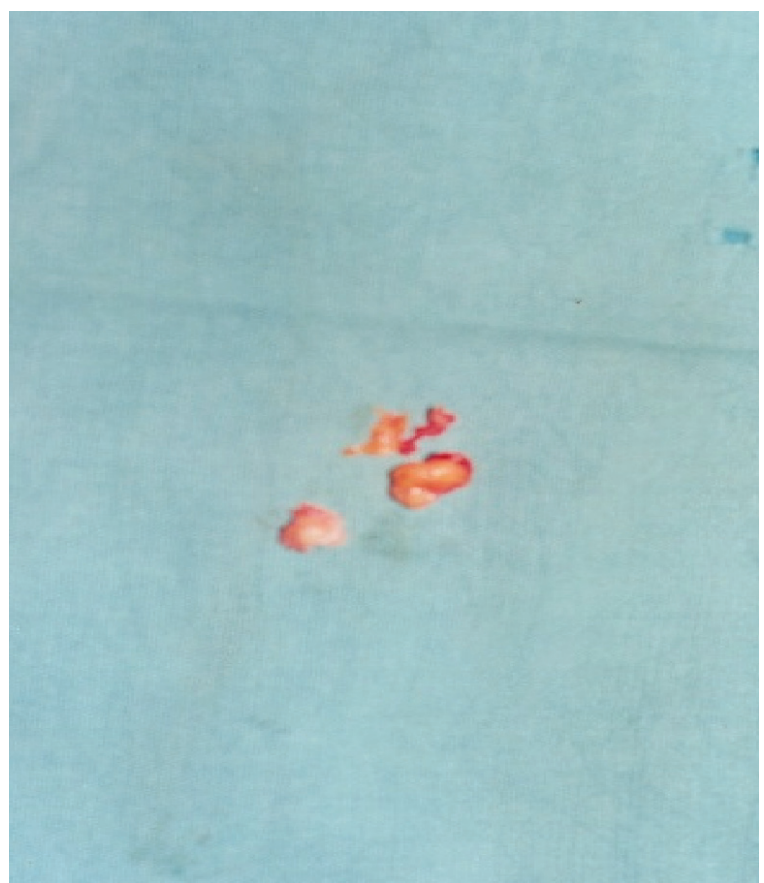

Fig 3b: Excised ossicles

effects, especially subcutaneous atrophy (15).

\section{Conclusions}

Osgood- Schlatter disease is a self-limiting condition. Conservative treatment (rest, analgesics, protecting braces) is associated with symptom resolution in a majority of cases. The presence of an ossicle may predict the need for surgery. Surgery when indicated has an excellent outcome.

\section{References}

1. Osgood, R B. Lessions of the tibial tubercle occurring during adolescence. Boston Med Surg. 1903. 148:114-7

2. Schlatter, C.. Verletzungen des Schnabel - fomigen fortsatzes der Oberon tibiaepiphyse. Beit Klin Chir. 1903. 38:874-7

3. Gholve PA, Scher DM, Khakharia S, et al. Osgood Schlatter syndrome. Curr Opin Pediatr. 2007; 19(1):44-50.

4. Bergami G, Barbuti D, Pezzoli F. Ultrasonographic findings in Osgood-Schlatter disease. Radiol Med(Torino), 1994; $88(4): 368$ 
5. Uhry E jr (1944) Osgood Sclatter's disease. Arch.Surg. 1944; 48:406-14

6. Lazarte GD, Rapp I H Pathogenesis of Osgood-Sclatter's disease. Am J pathology 1958; 34:803-15

7. Dunn J F Osgood-Schlatter disease. Am Fam Physician 1990: 41(1): 173-6

8. Sharad, W.J.W. Paediatric Orthopedics and fractures: Third edition. volume 2:749

9. Orava S, Malinen L, Karpakka J, et al. Results of surgical treatment of unresolved Osgood-Schlatter lesion. Ann Chir Gynaecol. 2000; 89(4):298-302.

10. Rosenberg Z S; kawelblumM; Cheung YY et al; Osgood Schlatter lesion: fracture or tendonitis? Scinitigraphic, CT, and MRI imaging features. Radiology 1992; 185(3):853-8
11. Binazzi R, Felli L, Vaccari V, Borelli P. Surgical treatment of unresolved Osgood-Schlatter lesion. Clin Orthop. Apr 1993; 202-4.

12. Krause BL, Williams JP, Catterall A. Natural history of Osgood-Schlatter disease. J Pediatr Orthop. 1990; 10(1):658.

13. Micheli LJ. The traction apophysitises. Clin Sports Med. 1987;6(2):389-404.

14. Flowers MJ, Bhadreshwar DR. Tibial tuberosity excision for symptomatic Osgood-Schlatter disease. J Pediatr Orthop. $1995 ; 15(3): 292-7$.

15. Rostron PK, Calver RF. Subcutaneous atrophy following methylprednisolone injection in Osgood-Schlatter epiphysitis. J Bone Joint Surg Am. 1979; 61(4):627-8. 